\title{
CASP8 -652 6N insertion/deletion polymorphism and overall cancer risk: evidence from 49 studies
}

\author{
Jiarong Cai ${ }^{1, *}$, Qingjian Ye ${ }^{2, *}$, Suling Luo ${ }^{3, *}$, Ze Zhuang ${ }^{4}$, Kui He ${ }^{5}$, Zhen-Jian Zhuo ${ }^{6}$, \\ Xiaochun Wan ${ }^{7,8}$ and Juan Cheng ${ }^{2}$ \\ ${ }^{1}$ Department of Urology, The Third Affiliated Hospital of Sun Yat-Sen University, Guangzhou 510630, China \\ ${ }^{2}$ Department of Gynecology, The Third Affiliated Hospital of Sun Yat-Sen University, Guangzhou 510630, China \\ ${ }^{3}$ Department of Otolaryngology, The First People's Hospital of Foshan (Affiliated Foshan Hospital of Sun Yat-Sen University), \\ Foshan 528000, China \\ ${ }^{4}$ Department of Joint Surgery and Orthopaedic Trauma, The Third Affiliated Hospital of Sun Yat-Sen University, Guangzhou \\ 510630, China \\ ${ }^{5}$ The Second People's Hospital of FuTian District, Shenzhen 518000, China \\ ${ }^{6}$ School of Chinese Medicine, Faculty of Medicine, The Chinese University of Hong Kong, Hong Kong 999077, China \\ ${ }^{7}$ Department of Pathology, Fudan University Shanghai Cancer Center, Shanghai 200032, China \\ ${ }^{8}$ Department of Oncology, Shanghai Medical College, Fudan University, Shanghai 200032, China \\ *These authors have contributed equally to this work \\ Correspondence to: Juan Cheng, email: mdchengjuan@sohu.com \\ Xiaochun Wan, email: wach126@163.com \\ Keywords: CASP8, -652 6N insertion/deletion, polymorphism, cancer risk, meta-analysis
}

Received: March 13, $2017 \quad$ Accepted: April 24, $2017 \quad$ Published: May 25, 2017

Copyright: Cai et al. This is an open-access article distributed under the terms of the Creative Commons Attribution License 3.0 (CC BY 3.0), which permits unrestricted use, distribution, and reproduction in any medium, provided the original author and source are credited.

\section{ABSTRACT}

The CASP8 -652 6N insertion/deletion (I/D) polymorphism reduces expression of caspase 8. We conducted a meta-analysis to clarify the relationship between this polymorphism and cancer risk. Eligible articles were retrieved from PubMed, EMBASE, CNKI, and WANFANG databases through February 2017. A total of 33 articles with 49 studies, including 33,494 cases and 36,397 controls, were analyzed. We found that the CASP8 $-6526 \mathrm{~N}$ ins/del polymorphism was associated with decreased overall cancer risk in five genetic models [DD vs. II: odds ratio $(O R)=0.76,95 \%$ confidence interval $(C I)=0.69-0.84$, ID vs. II: $\mathrm{OR}=0.87,95 \% \mathrm{CI}=0.83-0.92$, DD vs. ID/II: $\mathrm{OR}=0.82,95 \%$ $\mathrm{CI}=0.75-0.89$, ID/DD vs. II: $\mathrm{OR}=0.85,95 \% \mathrm{CI}=0.80-0.90$, and $\mathrm{D}$ vs. I: $\mathrm{OR}=0.87,95 \%$ $\mathrm{CI}=0.83-0.91]$. Stratified analyses showed that the polymorphism was associated with decreased risk of colorectal, breast, esophageal, renal cell, lung, cervical, bladder, gastric, and other cancers. Overall cancer risk was reduced in Asian and Caucasian patients, both hospital- and population-based studies, and both high and low quality studies. Our results highlight the role of the CASP8 $-6526 \mathrm{~N}$ ins/del polymorphism in decreasing cancer risk. Further studies with large-cohort populations, especially for specific cancer types and ethnic groups, are needed to confirm our findings.

\section{INTRODUCTION}

Cancer is a substantial public health burden worldwide and is the second leading cause of death in the United States. An estimated 1,688,780 new cancer cases and 600,920 cancer deaths will occur in the United States this year [1]. Approximately 14 million new cancer cases occurred worldwide in 2012, and by 2025, global cancer incidence is predicted to rise to 20 million new cases annually [2]. Although there are many cancer risk factors, genetic abnormalities play crucial roles in carcinogenesis [3-6].

Apoptosis is a control mechanism to prevent overproliferation in normal cells [7], and apoptosis pathway 
aberrations are implicated in cancer development [8]. Caspases are the main regulatory enzymes in the apoptosis pathway [9]. Caspase 8 mediates the extrinsic apoptosis pathway $[10,11]$. Human CASP8 is located on chromosome $2 \mathrm{q} 33 \sim \mathrm{q} 34$, has 11 exons [12], and is highly polymorphic with more than 474 single nucleotide polymorphisms (SNPs) according to the dbSNP database (http://www.ncbi.nlm.nih.gov/SNP). The CASP8 -652 $6 \mathrm{~N}$ ins/del polymorphism (rs3834129) is a six-nucleotide insertion/deletion variant located in the CASP8 promoter region [13], and leads to decreased CASP 8 expression. Impaired caspase 8 function reduces $\mathrm{T}$ lymphocyte "activation-induced cell death" (AICD) activity, which is important in immune surveillance of cancer cells [13].

Extensive epidemiological studies have assessed the association between the CASP8 $-6526 \mathrm{~N}$ ins/del polymorphism and cancer risk. However, these studies have not produced conclusive results. The most recent previous meta-analysis of this association, conducted in 2014 , assessed a relatively small number of studies. We performed this meta-analysis with a larger sample size to more precisely describe the association of interest.

\section{RESULTS}

\section{Study characteristics}

Our study selection workflow is shown in Figure 1. Our systematic computer-based search initially identified 108 potentially relevant articles. After scanning titles and abstracts, 67 articles about unrelated topics were excluded.
We further excluded 12 articles: eight were meta-analyses [14-21], three were case only studies [22-24], and one deviated from HWE [25]. Articles incorporating several ethnic groups or cancer types were separated into corresponding independent studies. In total, our analysis included datasets from 33 articles with 49 studies [13, 26-57].

Characteristics for 33,494 cases and 36,397 controls are summarized in Table 1. Of the included studies, 12 were conducted on colorectal cancer, nine on other cancers, eight on breast cancer, three on esophageal cancer, three on renal cell carcinoma, and two on lung cancer, cervical cancer, prostate cancer, bladder cancer, lymphoma cancer, and gastric cancer, respectively. Twenty-seven studies were conducted in Asians, 20 in Caucasians, one in Africans, and one in mixed populations. Twenty-four studies were of population-based design, 22 studies were of hospital-based design, and three did not mention study design in the original data. We also classified the studies as either low quality (25 studies) or high quality (24 studies) by quality score.

\section{Quantitative analysis}

Overall meta-analysis information is shown in Table 2 and Figure 2. In the pooled analysis, the CASP $8-6526 \mathrm{~N}$ ins/del polymorphism was associated with reduced overall cancer risk in all five genetic models (homozygous: DD vs. II: odds ratio $(\mathrm{OR})=0.76,95 \%$ confidence interval $(\mathrm{CI})=0.69-0.84$; heterozygous: ID vs. II: $\mathrm{OR}=0.87$, $95 \% \mathrm{CI}=0.83-0.92$; recessive: $\mathrm{DD}$ vs. $\mathrm{ID} / \mathrm{II}: \mathrm{OR}=0.82$,

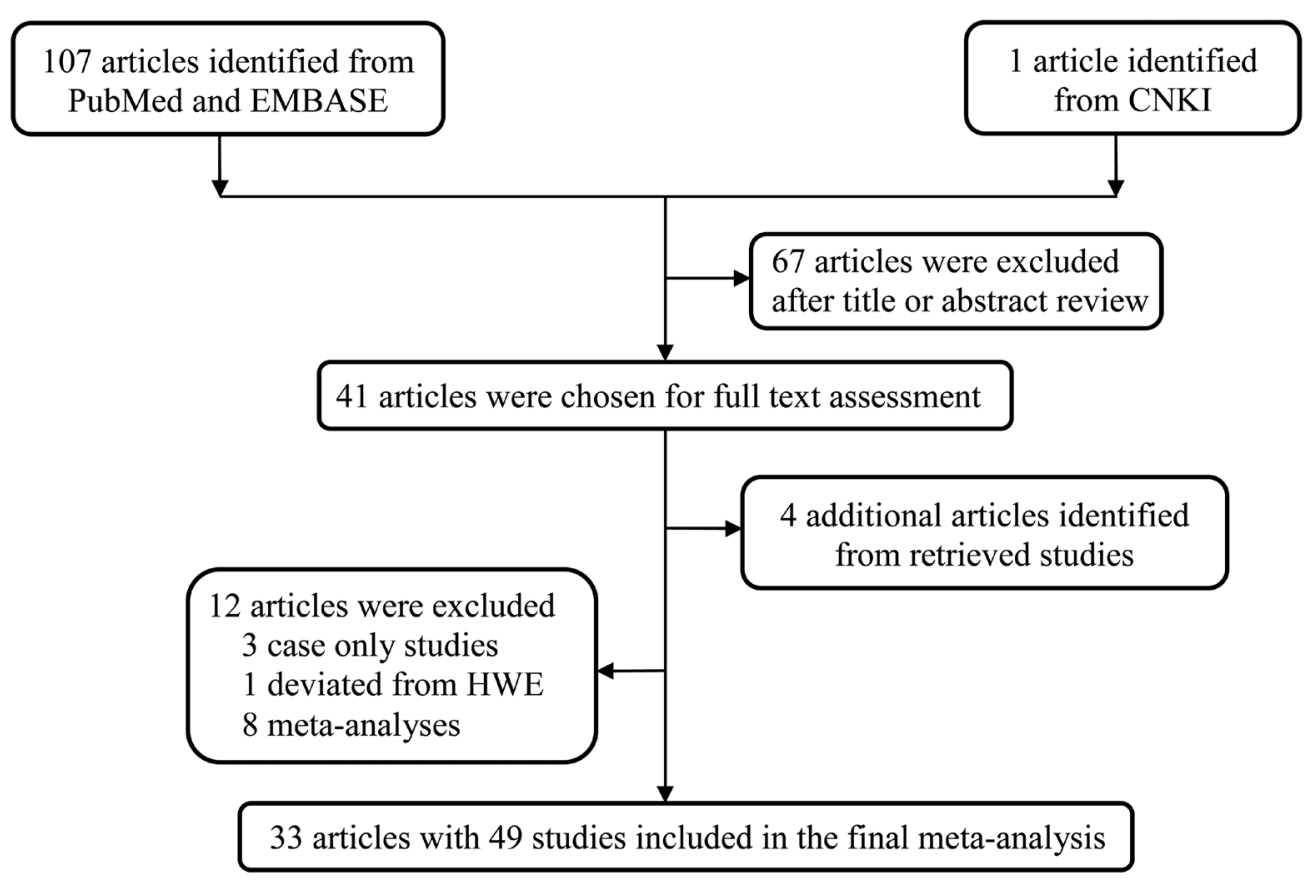

Figure 1: Flow diagram of the study selection process. 
Table 1: Characteristics of studies included in the meta-analysis

\begin{tabular}{|c|c|c|c|c|c|c|c|c|c|c|c|c|c|c|c|c|c|}
\hline \multirow{2}{*}{$\begin{array}{l}\text { Author last } \\
\text { name }\end{array}$} & \multirow{2}{*}{ Year } & \multirow{2}{*}{$\begin{array}{l}\text { Cancer } \\
\text { type }\end{array}$} & \multirow{2}{*}{ Country } & \multirow{2}{*}{ Ethnicity } & \multirow{2}{*}{ Design } & \multirow{2}{*}{$\begin{array}{l}\text { Genotype } \\
\text { method }\end{array}$} & \multicolumn{4}{|c|}{ Case } & \multicolumn{4}{|c|}{ Control } & \multirow{2}{*}{ MAF } & \multirow{2}{*}{ HWE } & \multirow{2}{*}{ Score } \\
\hline & & & & & & & II & ID & DD & All & II & ID & DD & All & & & \\
\hline Sun & 2007 & Lung & China & Asian & PB & PCR-RFLP & 756 & 348 & 45 & 1149 & 640 & 407 & 64 & 1111 & 0.24 & 0.947 & 11 \\
\hline Sun & 2007 & Esophagus & China & Asian & PB & PCR-RFLP & 652 & 328 & 38 & 1018 & 543 & 338 & 56 & 937 & 0.24 & 0.724 & 11 \\
\hline Sun & 2007 & Gastric & China & Asian & PB & PCR-RFLP & 262 & 142 & 16 & 420 & 233 & 152 & 25 & 410 & 0.25 & 0.975 & 11 \\
\hline Sun & 2007 & Colorectal & China & Asian & PB & PCR-RFLP & 605 & 280 & 33 & 918 & 528 & 304 & 58 & 890 & 0.24 & 0.116 & 11 \\
\hline Sun & 2007 & Breast & China & Asian & $\mathrm{PB}$ & PCR-RFLP & 699 & 371 & 49 & 1119 & 513 & 419 & 72 & 1004 & 0.28 & 0.279 & 11 \\
\hline Sun & 2007 & Cervical & China & Asian & PB & PCR-RFLP & 199 & 102 & 13 & 314 & 314 & 211 & 42 & 567 & 0.26 & 0.428 & 10 \\
\hline Yang & 2008 & Pancreatic & China & Asian & PB & PCR-RFLP & 268 & 111 & 18 & 397 & 521 & 323 & 63 & 907 & 0.25 & 0.185 & 13 \\
\hline Pittman & 2008 & Colorectal & England & Caucasian & PB & AS-PCR & 995 & 1897 & 987 & 3879 & 892 & 1872 & 897 & 3661 & 0.50 & 0.170 & 9 \\
\hline Frank & 2008 & Breast & Germany & Caucasian & HB & Fluorescent & 298 & 535 & 221 & 1054 & 270 & 506 & 263 & 1039 & 0.50 & 0.403 & 7 \\
\hline Frank & 2008 & Breast & England & Caucasian & PB & Fluorescent & 235 & 541 & 251 & 1027 & 245 & 608 & 321 & 1174 & 0.53 & 0.169 & 10 \\
\hline Frank & 2008 & Breast & Germany & Caucasian & PB & Fluorescent & 280 & 509 & 222 & 1011 & 285 & 492 & 229 & 1006 & 0.47 & 0.550 & 9 \\
\hline Frank & 2008 & Breast & England & Caucasian & $\mathrm{PB}$ & Fluorescent & 1133 & 2115 & 1050 & 4298 & 1149 & 2263 & 1062 & 4474 & 0.49 & 0.422 & 8 \\
\hline Cybulski & 2008 & Breast & Poland & Caucasian & $\mathrm{PB}$ & AS-PCR & 178 & 314 & 126 & 618 & 274 & 499 & 192 & 965 & 0.46 & 0.195 & 6 \\
\hline Cybulski & 2008 & Prostate & Poland & Caucasian & PB & AS-PCR & 139 & 236 & 110 & 485 & 274 & 499 & 192 & 965 & 0.46 & 0.195 & 6 \\
\hline $\mathrm{Li}$ & 2008 & Melanoma & USA & Caucasian & HB & PCR & 243 & 385 & 177 & 805 & 207 & 440 & 188 & 835 & 0.49 & 0.116 & 11 \\
\hline Wang & 2009 & Bladder & China & Asian & HB & PCR-RFLP & 238 & 115 & 12 & 365 & 205 & 138 & 25 & 368 & 0.26 & 0.786 & 10 \\
\hline Gangwar & 2009 & Bladder & India & Asian & HB & PCR-RFLP & 121 & 84 & 7 & 212 & 133 & 101 & 16 & 250 & 0.27 & 0.584 & 9 \\
\hline De Vecchi & 2009 & Breast & Italy & Caucasian & PB & PCR-RFLP & 162 & 301 & 117 & 580 & 106 & 206 & 94 & 406 & 0.49 & 0.752 & 7 \\
\hline Zhu & 2010 & $\mathrm{RCC}$ & China & Asian & HB & PCR-RFLP & 226 & 119 & 8 & 353 & 205 & 139 & 21 & 365 & 0.25 & 0.686 & 11 \\
\hline Srivastava & 2010 & Gallbladder & India & Asian & $\mathrm{PB}$ & PCR-RFLP & 147 & 69 & 12 & 228 & 122 & 84 & 24 & 230 & 0.29 & 0.103 & 11 \\
\hline Liu & 2010 & Colorectal & China & Asian & PB & PCR-RFLP & 233 & 116 & 21 & 370 & 528 & 278 & 32 & 838 & 0.20 & 0.538 & 13 \\
\hline $\mathrm{Li}$ & 2010 & HNSCC & USA & Caucasian & HB & PCR-RFLP & 311 & 456 & 256 & 1023 & 257 & 542 & 253 & 1052 & 0.50 & 0.324 & 10 \\
\hline Xiao & 2011 & Lymphoma & China & Asian & $\mathrm{NM}$ & PCR-PAGE & 43 & 17 & 4 & 64 & 89 & 38 & 6 & 133 & 0.19 & 0.460 & 3 \\
\hline Xiao & 2011 & Lymphoma & China & Asian & $\mathrm{NM}$ & PCR-PAGE & 49 & 23 & 3 & 75 & 63 & 40 & 4 & 107 & 0.22 & 0.442 & 3 \\
\hline Umar & 2011 & Esophageal & India & Asian & $\mathrm{PB}$ & PCR & 139 & 103 & 17 & 259 & 138 & 93 & 28 & 259 & 0.29 & 0.046 & 11 \\
\hline Theodoropoulos & 2011 & Colorectal & Greece & Caucasian & HB & RFLP-PCR & 103 & 201 & 98 & 402 & 120 & 254 & 106 & 480 & 0.49 & 0.194 & 9 \\
\hline Malik & 2011 & Esophageal & India & Asian & HB & RFLP-PCR & 68 & 59 & 8 & 135 & 96 & 75 & 24 & 195 & 0.32 & 0.127 & 8 \\
\hline Malik & 2011 & Gastric & India & Asian & HB & RFLP-PCR & 59 & 44 & 5 & 108 & 96 & 75 & 24 & 195 & 0.32 & 0.127 & 8 \\
\hline $\mathrm{Ma}$ & 2011 & Ovarian & China & Asian & HB & MassARRAY & 128 & 87 & 3 & 218 & 138 & 122 & 25 & 285 & 0.30 & 0.789 & 8 \\
\hline Liamarkopoulos & 2011 & Gastric & Greece & Caucasian & HB & PCR-RFLP & 35 & 42 & 11 & 88 & 120 & 254 & 106 & 480 & 0.49 & 0.194 & 7 \\
\hline Hart & 2011 & Lung & Norway & Caucasian & $\mathrm{PB}$ & TaqMan & 125 & 210 & 101 & 436 & 106 & 209 & 118 & 433 & 0.51 & 0.481 & 10 \\
\hline Chatterjee & 2011 & Cervical & $\begin{array}{l}\text { South } \\
\text { Africa }\end{array}$ & African & HB & PCR-RFLP & 18 & 63 & 25 & 106 & 43 & 129 & 85 & 257 & 0.58 & 0.614 & 6 \\
\hline $\mathrm{Fu}$ & 2011 & Prostate & China & Asian & HB & PCR-RFLP & 257 & 132 & 17 & 406 & 211 & 159 & 38 & 408 & 0.29 & 0.315 & 10 \\
\hline Wang & 2012 & $\mathrm{RCC}$ & China & Asian & HB & PCR-RFLP & 192 & 101 & 7 & 300 & 168 & 114 & 18 & 300 & 0.25 & 0.817 & 10 \\
\hline Wang & 2012 & PTC & China & Asian & HB & PCR-RFLP & 65 & 45 & 8 & 118 & 106 & 92 & 15 & 213 & 0.29 & 0.408 & 7 \\
\hline Tong & 2012 & ALL & China & Asian & HB & PCR-RFLP & 217 & 113 & 31 & 361 & 338 & 153 & 28 & 519 & 0.20 & 0.057 & 10 \\
\hline Hashemi & 2012 & Breast & Iran & Asian & HB & AS-PCR & 113 & 107 & 16 & 236 & 79 & 91 & 33 & 203 & 0.39 & 0.434 & 6 \\
\hline George & 2012 & Prostate & India & Asian & HB & PCR-RFLP & 84 & 69 & 12 & 165 & 116 & 83 & 6 & 205 & 0.23 & 0.050 & 9 \\
\hline Xiao & 2013 & Colorectal & China & Asian & HB & PCR-PAGE & 187 & 107 & 11 & 305 & 212 & 115 & 15 & 342 & 0.21 & 0.905 & 7 \\
\hline $\mathrm{Wu}$ & 2013 & Colorectal & China & Asian & HB & PCR-SSCP & 284 & 152 & 15 & 451 & 358 & 244 & 29 & 631 & 0.24 & 0.119 & 11 \\
\hline De Martino & 2013 & $\mathrm{RCC}$ & Austria & Caucasian & HB & PCR-RFLP & 72 & 138 & 40 & 250 & 53 & 129 & 68 & 250 & 0.53 & 0.572 & 9 \\
\hline Pardini & 2014 & Colorectal & Spain & Caucasian & PB & Taqman & 500 & 996 & 482 & 1978 & 425 & 802 & 420 & 1647 & 0.50 & 0.290 & 11 \\
\hline
\end{tabular}

(Continued) 


\begin{tabular}{|c|c|c|c|c|c|c|c|c|c|c|c|c|c|c|c|c|c|}
\hline \multirow{2}{*}{$\begin{array}{l}\text { Author last } \\
\text { name }\end{array}$} & \multirow{2}{*}{ Year } & \multirow{2}{*}{$\begin{array}{l}\text { Cancer } \\
\text { type }\end{array}$} & \multirow{2}{*}{ Country } & \multirow{2}{*}{ Ethnicity } & \multirow{2}{*}{ Design } & \multirow{2}{*}{$\begin{array}{l}\text { Genotype } \\
\text { method }\end{array}$} & \multicolumn{4}{|c|}{ Case } & \multicolumn{4}{|c|}{ Control } & \multirow{2}{*}{ MAF } & \multirow{2}{*}{ HWE } & \multirow{2}{*}{ Score } \\
\hline & & & & & & & II & ID & DD & All & II & ID & DD & All & & & \\
\hline Pardini & 2014 & Colorectal & Italy & Caucasian & PB & Taqman & 195 & 285 & 137 & 617 & 783 & 1230 & 538 & 2551 & 0.45 & 0.178 & 9 \\
\hline Pardini & 2014 & Colorectal & USA & Caucasian & PB & Taqman & 237 & 514 & 259 & 1010 & 383 & 794 & 403 & 1580 & 0.51 & 0.835 & 9 \\
\hline Pardini & 2014 & Colorectal & England & Caucasian & PB & Taqman & 410 & 825 & 341 & 1576 & 165 & 393 & 209 & 767 & 0.53 & 0.436 & 11 \\
\hline Pardini & 2014 & Colorectal & Czech & Caucasian & PB & Taqman & 239 & 479 & 249 & 967 & 169 & 326 & 177 & 672 & 0.51 & 0.443 & 10 \\
\hline Pardini & 2014 & Colorectal & Netherlands & Caucasian & PB & Taqman & 169 & 282 & 134 & 585 & 106 & 177 & 76 & 359 & 0.46 & 0.895 & 8 \\
\hline Tang & 2015 & OSCC & China & Asian & HB & PCR-RFLP & 328 & 159 & 18 & 505 & 276 & 197 & 34 & 507 & 0.26 & 0.885 & 10 \\
\hline Carvalho & 2015 & ALL & Brazil & Mixed & NM & PCR & 23 & 81 & 26 & 130 & 47 & 53 & 25 & 125 & 0.41 & 0.163 & 4 \\
\hline
\end{tabular}

MAF: minor allele frequency; HWE: Hardy-Weinberg equilibrium; OSCC: oral squamous cell carcinoma; PTC: papillary thyroid carcinoma; RCC: renal cell carcinoma; HNSCC: head and neck squamous cell carcinoma; ALL: acute lymphocytic leukemia; PB: population based; HB: hospital based; NM: not mentioned; PCR-PAGE: polymerase chain reaction-polyacrylamide gel electrophoresis; PCR-RFLP: polymerase chain reaction-restriction fragment length polymorphism; AS-PCR: allele-specific polymerase chain reaction.

Table 2: Meta-analysis of the association between the $C A S P 8-6526 \mathrm{~N}$ ins/del polymorphism and overall cancer risk

\begin{tabular}{|c|c|c|c|c|c|c|c|c|c|c|c|c|}
\hline \multirow{3}{*}{ Variables } & \multirow{3}{*}{$\begin{array}{l}\text { No. of } \\
\text { studies }\end{array}$} & \multirow{3}{*}{ Sample size } & \multirow{2}{*}{\multicolumn{2}{|c|}{$\frac{\text { Homozygous }}{\text { DD vs. II }}$}} & \multirow{2}{*}{\multicolumn{2}{|c|}{$\frac{\text { Heterozygous }}{\text { ID vs. II }}$}} & \multirow{2}{*}{\multicolumn{2}{|c|}{$\frac{\text { Recessive }}{\text { DD vs. ID/II }}$}} & \multirow{2}{*}{\multicolumn{2}{|c|}{$\frac{\text { Dominant }}{\text { ID/DD vs. II }}$}} & \multirow{2}{*}{\multicolumn{2}{|c|}{$\frac{\text { Allele }}{\text { D vs. I }}$}} \\
\hline & & & & & & & & & & & & \\
\hline & & & OR $(95 \% \mathrm{CI})$ & $P^{\text {het }}$ & OR (95\% CI) & $P^{\text {het }}$ & OR $(95 \% \mathrm{CI})$ & $P^{\text {het }}$ & OR (95\% CI) & $P^{\text {het }}$ & OR $(95 \% \mathrm{CI})$ & $P^{\text {het }}$ \\
\hline All & 49 & $33494 / 36397$ & $0.76(0.69-0.84)$ & $<0.001$ & $0.87(0.83-0.92)$ & $<0.001$ & $0.82(0.75-0.89)$ & $<0.001$ & $0.85(0.80-0.90)$ & $<0.001$ & $0.87(0.83-0.91)$ & $<0.001$ \\
\hline \multicolumn{13}{|l|}{ Cancer type } \\
\hline Colorectal & 12 & $13058 / 14418$ & $0.93(0.82-1.05)$ & 0.018 & $0.94(0.88-0.99)$ & 0.529 & $0.96(0.87-1.06)$ & 0.019 & $0.93(0.87-1.00)$ & 0.190 & $0.96(0.90-1.01)$ & 0.012 \\
\hline Breast & 8 & $9943 / 10271$ & $0.80(0.67-0.96)$ & 0.001 & $0.90(0.81-1.01)$ & 0.018 & $0.85(0.74-0.99)$ & 0.002 & $0.87(0.77-0.99)$ & 0.002 & $0.89(0.80-0.98)$ & $<0.001$ \\
\hline Esophageal & 3 & $1412 / 1196$ & $0.56(0.40-0.78)$ & 0.901 & $0.93(0.74-1.17)$ & 0.206 & $0.58(0.42-0.79)$ & 0.812 & $0.83(0.71-0.97)$ & 0.385 & $0.81(0.72-0.92)$ & 0.712 \\
\hline $\mathrm{RCC}$ & 3 & 903/915 & $0.39(0.26-0.59)$ & 0.852 & $0.78(0.64-0.95)$ & 0.998 & $0.46(0.32-0.66)$ & 0.732 & $0.71(0.58-0.86)$ & 0.949 & $0.70(0.61-0.82)$ & 0.966 \\
\hline Lung & 2 & $1585 / 1544$ & $0.66(0.51-0.87)$ & 0.473 & $0.75(0.64-0.88)$ & 0.385 & $0.75(0.59-0.95)$ & 0.458 & $0.73(0.63-0.85)$ & 0.453 & $0.78(0.69-0.87)$ & 0.273 \\
\hline Cervical & 2 & $420 / 824$ & $0.58(0.36-0.93)$ & 0.456 & $0.86(0.59-1.25)$ & 0.230 & $0.59(0.39-0.88)$ & 0.728 & $0.76(0.59-0.98)$ & 0.355 & $0.76(0.63-0.92)$ & 0.556 \\
\hline Prostate & 2 & $650 / 205$ & $1.54(0.67-3.55)$ & 0.100 & $0.99(0.79-1.23)$ & 0.411 & $1.50(0.74-3.07)$ & 0.135 & $1.05(0.85-1.29)$ & 0.321 & $1.11(0.93-1.33)$ & 0.255 \\
\hline Bladder & 2 & $577 / 618$ & $0.44(0.25-0.77)$ & 0.799 & $0.79(0.62-1.01)$ & 0.334 & $0.48(0.27-0.84)$ & 0.907 & $0.74(0.59-0.93)$ & 0.317 & $0.74(0.61-0.90)$ & 0.338 \\
\hline Lymphoma & 2 & $139 / 240$ & $1.19(0.44-3.23)$ & 0.729 & $0.82(0.52-1.31)$ & 0.635 & $1.26(0.47-3.39)$ & 0.789 & $0.86(0.56-1.34)$ & 0.559 & $0.93(0.64-1.35)$ & 0.535 \\
\hline Gastric & 2 & $196 / 675$ & $0.35(0.19-0.63)$ & 0.939 & $0.74(0.44-1.23)$ & 0.145 & $0.45(0.26-0.78)$ & 0.538 & $0.64(0.40-1.01)$ & 0.171 & $0.66(0.51-0.84)$ & 0.487 \\
\hline ALL & 2 & $491 / 644$ & $1.85(1.20-2.87)$ & 0.655 & $1.83(0.69-4.85)$ & 0.004 & $1.32(0.81-2.14)$ & 0.228 & $1.79(0.81-3.97)$ & 0.014 & $1.33(1.10-1.61)$ & 0.443 \\
\hline Others & 9 & $4120 / 4847$ & $0.57(0.43-0.75)$ & 0.009 & $0.72(0.65-0.79)$ & 0.976 & $0.65(0.49-0.88)$ & 0.001 & $0.70(0.64-0.77)$ & 0.855 & $0.75(0.68-0.84)$ & 0.013 \\
\hline \multicolumn{13}{|l|}{ Ethnicity } \\
\hline Asian & 27 & $10569 / 11219$ & $0.58(0.48-0.70)$ & $<0.001$ & $0.80(0.75-0.85)$ & 0.231 & $0.62(0.52-0.74)$ & 0.002 & $0.77(0.72-0.83)$ & 0.016 & $0.79(0.73-0.84)$ & $<0.001$ \\
\hline Caucasian & 20 & $22689 / 24796$ & $0.90(0.83-0.98)$ & 0.006 & $0.92(0.88-0.97)$ & 0.225 & $0.95(0.89-1.02)$ & 0.007 & $0.92(0.87-0.97)$ & 0.079 & $0.95(0.91-0.99)$ & 0.008 \\
\hline African & 1 & $106 / 257$ & $0.70(0.35-1.43)$ & I & $1.17(0.62-2.19)$ & l & $0.63(0.37-1.05)$ & I & $0.98(0.54-1.80)$ & I & $0.82(0.60-1.13)$ & / \\
\hline Mixed & 1 & $130 / 125$ & $2.13(1.01-4.46)$ & I & $3.12(1.70-5.73)$ & l & $1.00(0.54-1.85)$ & l & $2.80(1.57-5.00)$ & l & $1.50(1.05-2.12)$ & I \\
\hline \multicolumn{13}{|c|}{ Source of control } \\
\hline PB & 24 & $25259 / 26848$ & $0.83(0.75-0.92)$ & $<0.001$ & $0.89(0.84-0.94)$ & 0.008 & $0.89(0.82-0.96)$ & $<0.001$ & $0.87(0.81-0.93)$ & $<0.001$ & $0.89(0.85-0.95)$ & $<0.001$ \\
\hline HB & 22 & $7966 / 9184$ & $0.61(0.49-0.75)$ & $<0.001$ & $0.83(0.77-0.89)$ & 0.213 & $0.67(0.55-0.82)$ & $<0.001$ & $0.79(0.73-0.87)$ & 0.024 & $0.81(0.75-0.88)$ & $<0.001$ \\
\hline NM & 3 & $269 / 365$ & $1.73(0.95-3.14)$ & 0.619 & $1.30(0.53-3.20)$ & 0.003 & $1.07(0.63-1.80)$ & 0.896 & $1.29(0.58-2.88)$ & 0.005 & $1.14(0.79-1.64)$ & 0.156 \\
\hline \multicolumn{13}{|l|}{ Quality score } \\
\hline$>9$ & 24 & $16745 / 16831$ & $0.67(0.58-0.77)$ & $<0.001$ & $0.81(0.76-0.87)$ & 0.008 & $0.75(0.66-0.85)$ & $<0.001$ & $0.78(0.73-0.84)$ & $<0.001$ & $0.81(0.76-0.87)$ & $<0.001$ \\
\hline$\leq 9$ & 25 & $16749 / 19566$ & $0.87(0.77-0.99)$ & $<0.001$ & $0.95(0.90-1.01)$ & 0.289 & $0.90(0.81-1.00)$ & $<0.001$ & $0.94(0.88-1.00)$ & 0.048 & $0.94(0.90-0.99)$ & $<0.001$ \\
\hline
\end{tabular}

Values were in bold, if the $95 \%$ CI excluded 1 or $P<0.05$

Het: heterogeneity; RCC: renal cell carcinoma; ALL: acute lymphocytic leukemia; HB: hospital based; PB: population based; NM: not mentioned. 
95\% $\mathrm{CI}=0.75-0.89$; dominant: ID/DD vs. II: $\mathrm{OR}=0.85$, $95 \% \mathrm{CI}=0.80-0.90$; and allele: $\mathrm{D}$ vs. $\mathrm{I}: \mathrm{OR}=0.87,95 \%$ $\mathrm{CI}=0.83-0.91$.

In cancer type stratification analysis, the CASP8 $-6526 \mathrm{~N}$ ins/del polymorphism decreased risk for colorectal cancer, breast cancer, esophageal cancer, renal cell carcinoma, lung cancer, cervical cancer, bladder cancer, gastric cancer, and other cancers. However, acute lymphocytic leukemia risk was increased (DD vs. II:
$\mathrm{OR}=1.85,95 \% \mathrm{CI}=1.20-2.87$; and $\mathrm{D}$ vs. $\mathrm{I}: \mathrm{OR}=1.33,95 \%$ $\mathrm{CI}=1.10-1.61)$. We observed no correlations between the CASP $8-6526 \mathrm{~N}$ ins/del polymorphism and prostate cancer or lymphoma.

Stratification analysis by ethnicity revealed a decreased cancer risk for Asians (DD vs. II: $\mathrm{OR}=0.58$, 95\% CI $=0.48-0.70$ ) and Caucasians (DD vs. II: $\mathrm{OR}=0.90$, $95 \% \mathrm{CI}=0.83-0.98)$, and an increased risk in mixed populations (DD vs. II: $\mathrm{OR}=2.13,95 \% \mathrm{CI}=1.01-4.46$ ). We

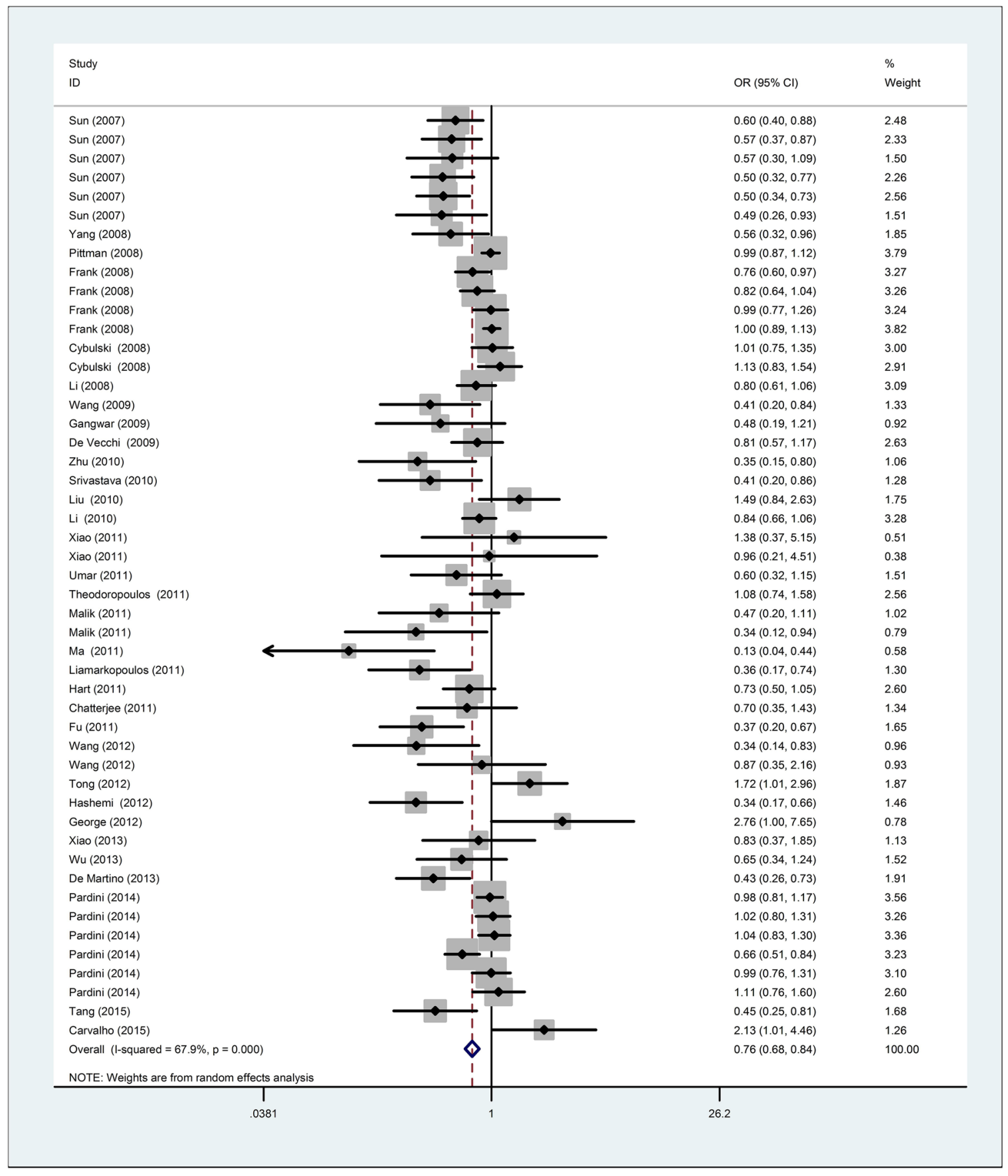

Figure 2: Forest plot of the association between the CASP8 $-6526 \mathrm{~N}$ ins/del polymorphism and cancer risk via the homozygous model. The OR and 95\% CI for each study are plotted as a box and horizontal line. $\diamond$, pooled ORs and the corresponding $95 \%$ CIs. 
also found that the CASP $-6526 \mathrm{~N}$ ins/del polymorphism decreased cancer risk in population-based (DD vs. II: $\mathrm{OR}=0.83,95 \% \mathrm{CI}=0.75-0.92)$ and hospital-based groups (DD vs. II: $\mathrm{OR}=0.61,95 \% \mathrm{CI}=0.49-0.75$ ). Similarly, the CASP8 $-6526 \mathrm{~N}$ ins/del polymorphism was associated with decreased cancer risk in both the high quality (DD vs. II: $\mathrm{OR}=0.67,95 \% \mathrm{CI}=0.58-0.77)$ and low quality study groups (DD vs. II: $\mathrm{OR}=0.87,95 \% \mathrm{CI}=0.77-0.99$ ).

\section{Heterogeneity and sensitivity analysis}

Heterogeneity was observed in all five genetic models $(P<0.001$, Q test). Therefore, the random-effect model was adopted to generate ORs and 95\% CIs. We also conducted a sequential leave-one-out sensitivity analysis to evaluate the impact of a single study on the pooled estimates. Omission of no single study influenced the pooled ORs, indicating the statistical robustness of this meta-analysis (data not shown).

\section{Publication bias}

Begg's funnel plot shapes did not suggest any obvious asymmetry (Figure 3). Egger's test results (DD vs. II: $\mathrm{t}=-4.17, P<0.001$; ID vs. II: $\mathrm{t}=-0.12, P=0.905$; DD vs. ID/II: $\mathrm{t}=-1.15, P=0.257$; ID/DD vs. II: $\mathrm{t}=-1.09, P=0.281$; and $\mathrm{D}$ vs. $\mathrm{I}: \mathrm{t}=-3.33, P=0.002$ ) suggested that publication bias existed in the homozygote and allele models.

\section{Trial sequential analysis}

To minimize random errors and strengthen the robustness of our conclusions, we performed trial sequential analysis (TSA) (Figure 4). The cumulative Z-curve crossed the trial sequential monitoring boundary before the required information size was reached, suggesting that our study conclusion was convincing and no additional evidence was needed to verify said conclusion.

\section{DISCUSSION}

The present meta-analysis comprehensively evaluated the relationship between the CASP8 $-6526 \mathrm{~N}$ ins/del polymorphism and cancer risk across 49 studies (33,494 cases and 36,397 controls). The CASP8 -652 $6 \mathrm{~N}$ ins/del polymorphism was associated with decreased cancer risk in all five genetic models, and in the following subgroups: colorectal cancer, breast cancer, esophageal cancer, renal cell carcinoma, lung cancer, cervical cancer, bladder cancer, gastric cancer, other cancers, Asian, Caucasian, mixed population, population-based controls, hospital-based controls, high quality score, and low quality score.

Human immune cells play critical roles in eliminating potentially malignant cells [58]. Caspase 8

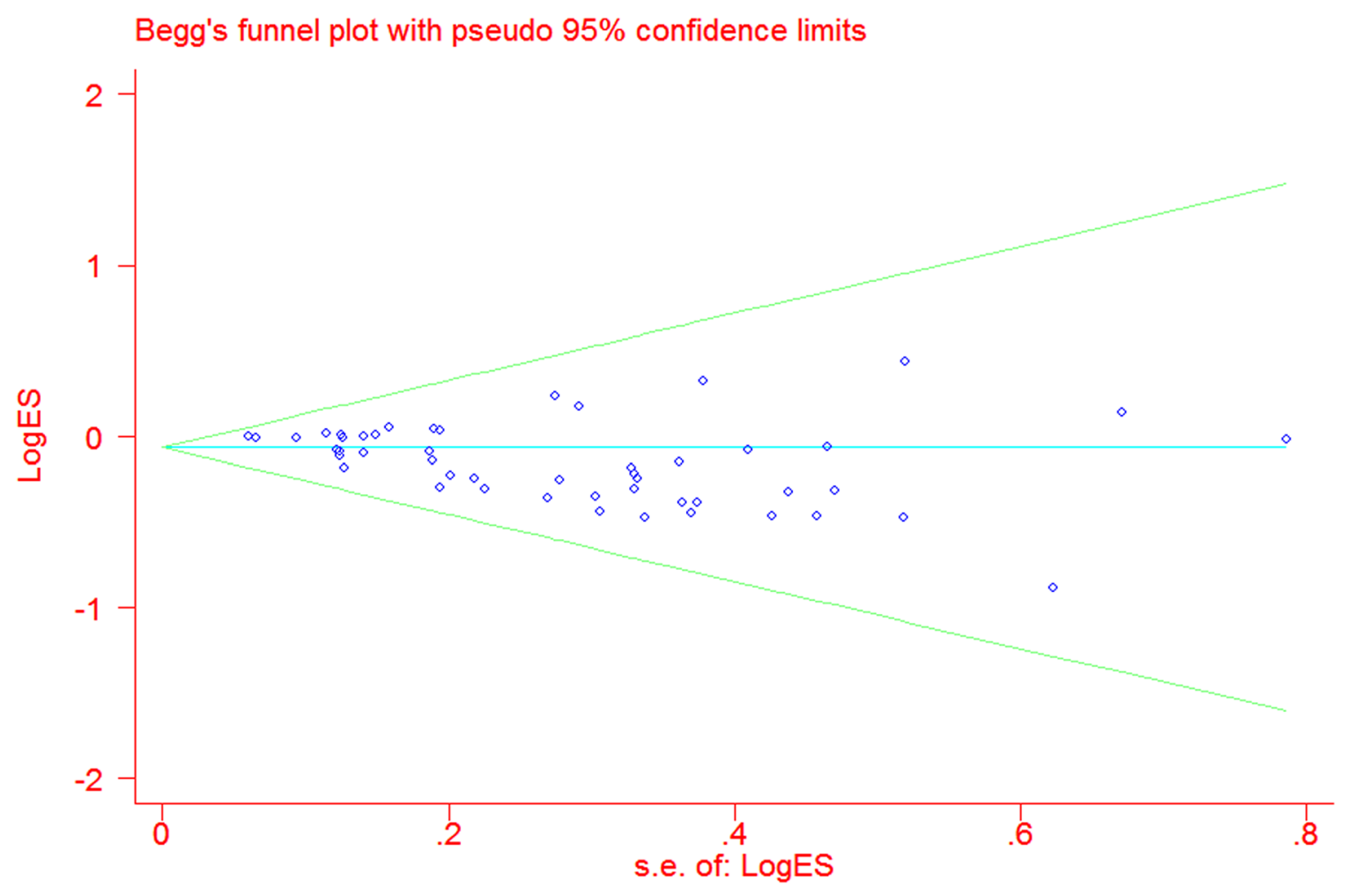

Figure 3: Funnel plot analysis to detect publication bias for the CASP8 $-6526 \mathrm{~N}$ ins/del polymorphism via the homozygous model. Each point represents a separate study for the indicated association. 
protein (encoded by CASP8) maintains immune cells by mediating the activation-apoptosis balance [59]. Low caspase 8 expression or functional aberrations may decrease $\mathrm{T}$ lymphocyte apoptotic reactivity [13]. The CASP8 $-6526 \mathrm{~N}$ del variant inactivates the transcription factor stimulatory protein 1 binding site, decreasing CASP8 transcription [13]. Thus, this variant may affect cancer susceptibility by influencing immune surveillance.

The first case-control study of the CASP $8-652$ $6 \mathrm{~N}$ del variant-cancer association, with 4,995 cases and 4,972 controls, was conducted by Sun, et al. in 2007 [13]. The authors found that the CASP8 -652 6N deletion allele decreased susceptibility to lung, colorectal, esophageal, breast, cervical, and gastric cancers. Biochemical assays illustrated that this variant might decrease apoptotic reactivity in cancer cell-stimulated $\mathrm{T}$ lymphocytes. However, Umar, et al. did not detect any association between the CASP8 $-6526 \mathrm{~N}$ polymorphism and esophageal squamous cell carcinoma (ESCC) risk in 259 patients and 259 healthy controls in an Indian population [45]. Several meta-analyses have attempted to address these contradictory conclusions. A 2012 metaanalysis by Chen, et al., including 19 case-control studies with 23,172 cases and 26,532 controls, associated the del allele, ins/del genotype, and del allele carriers with reduced overall cancer risk [16]. Similarly, in a metaanalysis incorporating 11 reports with 27,459 cases and 31,614 controls, Yin, et al. associated the CASP8 -652 $5 \mathrm{~N}$ del polymorphism with reduced overall cancer risk via homozygous, dominant, and recessive models [15]. In 2014, breast cancer- and colorectal cancer-specific meta-analyses $[19,20]$ concluded that the CASP8 -652
$6 \mathrm{~N}$ del polymorphism reduced cancer risk. However, no association was observed between this polymorphism and prostate cancer susceptibility in a meta-analysis by Zhang, et al. [21].

To provide a more robust clarification, our metaanalysis included all eligible studies published in either the English or Chinese language. In agreement with the four previously published meta-analyses, we found that the CASP $8-6526 \mathrm{~N}$ ins/del polymorphism was associated with reduced overall cancer risk. In subgroup analyses, the polymorphism was associated with reduced risk of colorectal cancer, breast cancer, esophageal cancer, renal cell carcinoma, lung cancer, cervical cancer, bladder cancer, gastric cancer, and other cancers, but not prostate cancer or lymphoma. A prostate cancer-specific metaanalysis also failed to detect a significant association. This may be attributed to cancer-specific inherent heterogeneity $[60,61]$. Additionally, we observed an association with decreased cancer risk among Asians and Caucasians, but not Africans or mixed ethnicity populations. However, the limited number of studies in Africans and mixed ethnicity population may account for this finding, and CASP $8-652$ $6 \mathrm{~N}$ ins/del polymorphism allelic distributions might vary geographically and ethnically.

Our meta-analysis of the association between the CASP $-6526 \mathrm{~N}$ ins/del polymorphism and cancer risk is by far the largest such meta-analysis with the greatest statistical power published thus far. We conducted subgroup analyses to provide a more precise, cancer type-specific conclusion, and we assessed studies in both Chinese and English to minimize selection bias. However, our study had certain limitations. First, for

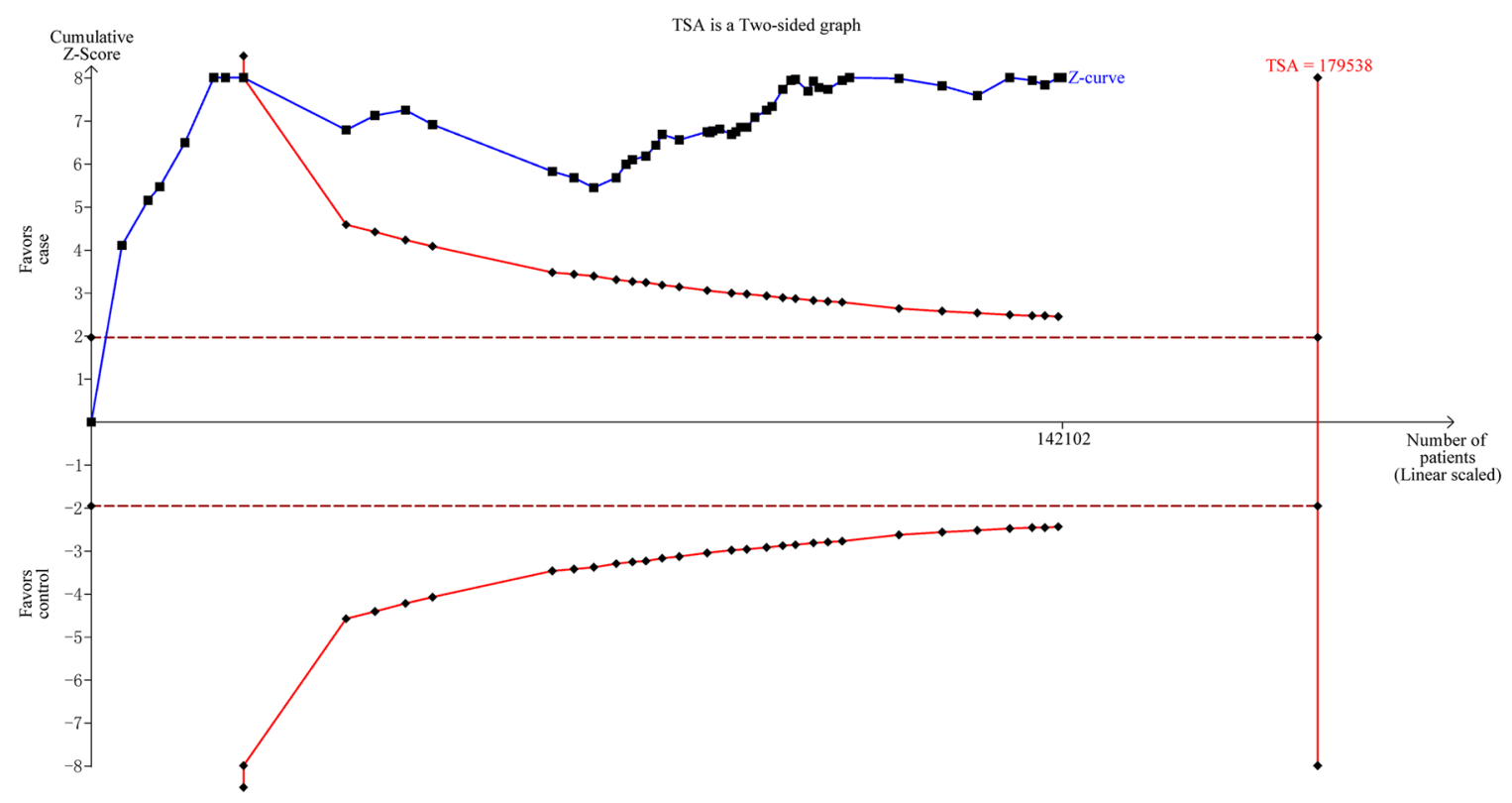

Figure 4: Trial sequential analysis for the $C A S P 8-6526 \mathrm{~N}$ ins/del polymorphism via the allele contrast model. 
some types of cancers, the calculated association was not robust enough due to limited numbers of original studies. Second, only one CASP 8 genetic variant was considered, and confounding factors, such as other genetic mutations and environmental exposures, also influence cancer susceptibility. Third, the observed between-study heterogeneity may reduce the validity of our conclusions. Finally, publication bias, language bias, or selection bias might lead to false positive or negative findings.

The present work robustly concludes that the CASP8 $-6526 \mathrm{~N}$ ins/del polymorphism is associated with reduced overall cancer risk. Refined studies with larger sample sizes, especially for certain cancer types and ethnic groups, are needed to fully validate this relationship.

\section{MATERIALS AND METHODS}

\section{Search strategy}

We conducted a literature search in PubMed and EMBASE using the following combined terms: 'Caspase 8 ' or 'CASP8' and 'polymorphism' or 'polymorphisms' or 'single nucleotide polymorphism' or 'SNP' or 'variant' and 'cancer' or 'tumor' or 'carcinoma' or 'carcinogenesis' or 'neoplasm'. We also searched studies written in Chinese from two databases, WANFANG and CNKI. We searched for articles published through February 2017 without imposing language limitations. Relevant references were also collected from retrieved articles. Only the largest or the most recent study was retained if studies contained overlapping data.

\section{Inclusion/exclusion criteria}

Studies included in our analysis met the following criteria: (1) evaluated CASP8 $-6526 \mathrm{~N}$ ins/del polymorphism with respect to cancer risk; (2) case-control design; (3) sufficient information to extract genotype frequencies for all subjects; (4) genotype frequency of controls consistent with Hardy-Weinberg equilibrium (HWE); (5) publication language was English or Chinese. Criteria for exclusion included: (1) abstract only, review, or meta-analysis; (2) case only studies; (3) no detailed genotyping data provided; (4) repeated publication.

\section{Data extraction}

Two authors (Jiarong Cai and Qingjian Ye) independently identified all eligible studies, and extracted data was included in the meta-analysis following consensus. The following items were recorded from each study: first author's name, year of publication, country, patient ethnicity, cancer type, source of controls, genotyping method, and genotype distributions of cases and controls. If reports contained more than one ethnic group or cancer type, we separated them into different studies.

\section{Trial sequential analysis}

After adopting a risk of 5\% for type I errors and $30 \%$ for type II errors, the required information size (sample sizes from all included trials) was calculated. TSA monitoring boundaries were built based on required information size and risk for type I and type II errors. If the cumulative Z-curve crossed the TSA monitoring boundary before the required information size was reached (i.e. if a sufficiently small $P$-value was achieved), further trials were unnecessary.

\section{Statistical analyses}

We used the Chi-square test to ensure that all control genotype frequencies were in agreement with HWE. Odds ratios (ORs) with corresponding 95\% confidence intervals (CIs) obtained from case and control genotype frequencies were used to assess the strength of association between the CASP8 $-6526 \mathrm{~N}$ ins/del polymorphism and cancer risk. Pooled ORs were calculated for the following five genetic models: homozygote model (DD vs. II), heterozygote model (ID vs. II), recessive model (DD vs. ID/II), dominant model (ID/DD vs. II), and allele model (D vs. I). The Cochran's Chi-square-based Q-test and the inconsistency index $\left(\mathrm{I}^{2}\right.$ statistics) were adopted to assess heterogeneity between study results. $\mathrm{I}^{2}<50 \%$ or $P>0.10$ indicates heterogeneity. The fixed-effects model (MantelHaenszel method) was used to estimate the pooled OR if no heterogeneity existed $\left(\mathrm{I}^{2}<50 \%\right.$ or $\left.P>0.10\right)$. Otherwise, the random-effects model (DerSimonian and Laird method) was applied. Quality assessment for each study was performed using the quality assessment criteria described previously (Supplementary Table 1) [62-65]. To decrease heterogeneity among studies, we conducted stratification analyses by ethnicity, cancer type, control source, and quality score. By adopting one-way sensitivity analysis, we recalculated the pooled ORs to assess the robustness of the results. We also conducted Begg's funnel plot and Egger's regression asymmetry test to examine potential publication bias [66-69]. STATA software v. 11.0 (Stata Corporation, College Station, TX) was used for statistical analyses [70]. $P<0.05$ (two-sided) was considered statistically significant.

\section{CONFLICTS OF INTEREST}

The authors declare no conflicts of interest.

\section{GRANT SUPPORT}

This study was supported by grants from the National Natural Science Foundation of China (Grant No. 31172044). 


\section{REFERENCES}

1. Siegel RL, Miller KD, Jemal A. Cancer statistics, 2017. CA Cancer J Clin. 2017; 67:7-30.

2. Ferlay J, Soerjomataram I, Dikshit R, Eser S, Mathers C, Rebelo M, Parkin DM, Forman D, Bray F. Cancer incidence and mortality worldwide: sources, methods and major patterns in GLOBOCAN 2012. Int J Cancer. 2015; 136:E359-386.

3. Pharoah PD, Dunning AM, Ponder BA, Easton DF. Association studies for finding cancer-susceptibility genetic variants. Nat Rev Cancer. 2004; 4:850-860.

4. Foulkes WD. Inherited susceptibility to common cancers. N Engl J Med. 2008; 359:2143-2153.

5. Wang Y, Wu XS, He J, Ma T, Lei W, Shen ZY. A novel TP53 variant (rs78378222 A $>$ C) in the polyadenylation signal is associated with increased cancer susceptibility: evidence from a meta-analysis. Oncotarget. 2016; 7:32854-32865. doi: 10.18632/oncotarget.9056.

6. Xue W, Zhu M, Wang Y, He J, Zheng L. Association between PLCE1 rs2274223 A > G polymorphism and cancer risk: proof from a meta-analysis. Sci Rep. 2015; 5:7986.

7. Hengartner MO. The biochemistry of apoptosis. Nature. 2000; 407:770-776.

8. Thompson CB. Apoptosis in the pathogenesis and treatment of disease. Science. 1995; 267:1456-1462.

9. Nunez G, Benedict MA, Hu Y, Inohara N. Caspases: the proteases of the apoptotic pathway. Oncogene. 1998; 17:3237-3245.

10. Danial NN, Korsmeyer SJ. Cell death: critical control points. Cell. 2004; 116:205-219.

11. Feltham R, Vince JE, Lawlor KE. Caspase-8: not so silently deadly. Clin Transl Immunology. 2017; 6:e124.

12. Grenet J, Teitz T, Wei T, Valentine V, Kidd VJ. Structure and chromosome localization of the human CASP8 gene. Gene. 1999; 226:225-232.

13. Sun T, Gao Y, Tan W, Ma S, Shi Y, Yao J, Guo Y, Yang M, Zhang X, Zhang Q, Zeng C, Lin D. A six-nucleotide insertion-deletion polymorphism in the CASP8 promoter is associated with susceptibility to multiple cancers. Nat Genet. 2007; 39:605-613.

14. Sergentanis TN, Economopoulos KP. Association of two CASP8 polymorphisms with breast cancer risk: a metaanalysis. Breast Cancer Res Treat. 2010; 120:229-234.

15. Yin M, Yan J, Wei S, Wei Q. CASP8 polymorphisms contribute to cancer susceptibility: evidence from a metaanalysis of 23 publications with 55 individual studies. Carcinogenesis. 2010; 31:850-857

16. Chen D, Ma T, Liu XW, Liu Z. CASP-8 $-6526 \mathrm{~N}$ ins/del polymorphism and cancer risk: a literature-based systematic HuGE review and meta-analysis. Exp Ther Med. 2012; 4:762-770.
17. Zhang F, Yang Y, Guo C, Wang Y. CASP8 -652 6N del polymorphism and cancer risk: a meta-analysis of 30 casecontrol studies in 50,112 subjects. Mutagenesis. 2012; 27:559-566.

18. Zhang YJ, Zhong XP, Chen Y, Liu SR, Wu G, Liu YF. Association between CASP-8 gene polymorphisms and cancer risk in some Asian population based on a HuGE review and meta-analysis. Genet Mol Res. 2013; 12:6466-6476.

19. Li Z, Wang J, Wang F, Ma Z, Yu Z. CAS P8 $-6526 \mathrm{~N}$ del polymorphism and breast cancer risk: a systematic review and meta-analysis. Neth J Med. 2014; 72:10-16.

20. Peng Q, Lao X, Tang W, Chen Z, Li R, Wang J, Deng Y, Li T, Qin X, Li S. CASP8 $-6526 \mathrm{~N}$ del polymorphism contributes to colorectal cancer susceptibility: evidence from a meta-analysis. PLoS One. 2014; 9:e87925.

21. Zhang CD, Li HT, Liu K, Lin ZD, Peng QL, Qin X, He M, $\mathrm{Wu}$ H, Mo ZN, Yang XL. Impact of caspase-8 (CASP8) $-6526 \mathrm{~N}$ del and D302H polymorphisms on prostate cancer in different ethnic groups. Asian Pac J Cancer Prev. 2014; 15:7713-7718.

22. Yoo SS, Choi JE, Lee WK, Choi YY, Kam S, Kim MJ, Jeon HS, Lee EB, Kim DS, Lee MH, Kim IS, Jheon S, Park JY. Polymorphisms in the CASPASE genes and survival in patients with early-stage non-small-cell lung cancer. J Clin Oncol. 2009; 27:5823-5829.

23. Choi JY, Kim JG, Lee YJ, Chae YS, Sohn SK, Moon JH, Kang BW, Jung MK, Jeon SW, Park JS, Choi GS. Prognostic impact of polymorphisms in the CASPASE genes on survival of patients with colorectal cancer. Cancer Res Treat. 2012; 44:32-36.

24. Kuhlmann JD, Bankfalvi A, Schmid KW, Callies R, Kimmig R, Wimberger P, Siffert W, Bachmann HS. Prognostic relevance of caspase $8-652$ 6N InsDel and Asp302His polymorphisms for breast cancer. BMC Cancer. 2016; 16:618.

25. Kesarwani P, Mandal RK, Maheshwari R, Mittal RD. Influence of caspases 8 and 9 gene promoter polymorphism on prostate cancer susceptibility and early development of hormone refractory prostate cancer. BJU Int. 2011; 107:471-476.

26. Cybulski C, Wokolorczyk D, Gliniewicz B, Sikorski A, Gorski B, Jakubowska A, Huzarski T, Byrski T, Debniak T, Gronwald J, Lubinski J, Narod SA. A six-nucleotide deletion in the CASP8 promoter is not associated with a susceptibility to breast and prostate cancers in the Polish population. Breast Cancer Res Treat. 2008; 112:367-368.

27. Frank B, Rigas SH, Bermejo JL, Wiestler M, Wagner K, Hemminki K, Reed MW, Sutter C, Wappenschmidt B, Balasubramanian SP, Meindl A, Kiechle M, Bugert P, et al. The CASP8 -652 6N del promoter polymorphism and breast cancer risk: a multicenter study. Breast Cancer Res Treat. 2008; 111:139-144. 
28. Li C, Zhao H, Hu Z, Liu Z, Wang LE, Gershenwald JE, Prieto VG, Lee JE, Duvic M, Grimm EA, Wei Q. Genetic variants and haplotypes of the caspase- 8 and caspase- 10 genes contribute to susceptibility to cutaneous melanoma. Hum Mutat. 2008; 29:1443-1451.

29. Pittman AM, Broderick P, Sullivan K, Fielding S, Webb E, Penegar S, Tomlinson I, Houlston RS. CASP8 variants $\mathrm{D} 302 \mathrm{H}$ and $-6526 \mathrm{~N}$ ins/del do not influence the risk of colorectal cancer in the United Kingdom population. Br J Cancer. 2008; 98:1434-1436.

30. Yang M, Sun T, Wang L, Yu D, Zhang X, Miao X, Liu J, Zhao D, Li H, Tan W, Lin D. Functional variants in cell death pathway genes and risk of pancreatic cancer. Clin Cancer Res. 2008; 14:3230-3236.

31. De Vecchi G, Verderio P, Pizzamiglio S, Manoukian S, Barile M, Fortuzzi S, Ravagnani F, Pierotti MA, Radice P, Peterlongo P. Evidences for association of the CASP8 $-6526 \mathrm{~N}$ del promoter polymorphism with age at diagnosis in familial breast cancer cases. Breast Cancer Res Treat. 2009; 113:607-608.

32. Gangwar R, Mandhani A, Mittal RD. Caspase 9 and caspase 8 gene polymorphisms and susceptibility to bladder cancer in north Indian population. Ann Surg Oncol. 2009; 16:2028-2034.

33. Wang M, Zhang Z, Tian Y, Shao J, Zhang Z. A sixnucleotide insertion-deletion polymorphism in the CASP8 promoter associated with risk and progression of bladder cancer. Clin Cancer Res. 2009; 15:2567-2572.

34. Li C, Lu J, Liu Z, Wang LE, Zhao H, El-Naggar AK, Sturgis EM, Wei Q. The six-nucleotide deletion/insertion variant in the CASP8 promoter region is inversely associated with risk of squamous cell carcinoma of the head and neck. Cancer Prev Res (Phila). 2010; 3:246-253.

35. Liu B, Zhang Y, Jin M, Ni Q, Liang X, Ma X, Yao K, Li Q, Chen K. Association of selected polymorphisms of CCND1, p21, and caspase8 with colorectal cancer risk. Mol Carcinog. 2010; 49:75-84.

36. Srivastava K, Srivastava A, Mittal B. Caspase- 8 polymorphisms and risk of gallbladder cancer in a northern Indian population. Mol Carcinog. 2010; 49:684-692.

37. Zhu J, Qin C, Wang M, Yan F, Ju X, Meng X, Ding Q, Li P, Yang J, Cao Q, Zhang Z, Yin C. Functional polymorphisms in cell death pathway genes and risk of renal cell carcinoma. Mol Carcinog. 2010; 49:810-817.

38. Chatterjee K, Williamson AL, Hoffman M, Dandara C. CASP8 promoter polymorphism is associated with highrisk HPV types and abnormal cytology but not with cervical cancer. J Med Virol. 2011; 83:630-636.

39. Fu G, Tang J, Wang M, Qin C, Yan F, Ding Q, Yin C, Wang $\mathrm{X}$, Zhang Z. CASP8 promoter polymorphism, mRNA expression and risk of prostate cancer among Chinese men. J Biomed Res. 2011; 25:128-134.

40. Hart K, Landvik NE, Lind H, Skaug V, Haugen A, Zienolddiny S. A combination of functional polymorphisms in the CASP8, MMP1, IL10 and SEPS1 genes affects risk of non-small cell lung cancer. Lung Cancer. 2011; 71:123-129.

41. Liamarkopoulos E, Gazouli M, Aravantinos G, Tzanakis N, Theodoropoulos G, Rizos S, Nikiteas N. Caspase 8 and caspase 9 gene polymorphisms and susceptibility to gastric cancer. Gastric Cancer. 2011; 14:317-321.

42. Ma X, Zhang J, Liu S, Huang Y, Chen B, Wang D. Polymorphisms in the CASP8 gene and the risk of epithelial ovarian cancer. Gynecol Oncol. 2011; 122:554-559.

43. Malik MA, Zargar SA, Mittal B. A six-nucleotide deletion polymorphism in the casp8 promoter is associated with reduced risk of esophageal and gastric cancers in Kashmir valley. Indian J Hum Genet. 2011; 17:152-156.

44. Theodoropoulos GE, Gazouli M, Vaiopoulou A, Leandrou M, Nikouli S, Vassou E, Kouraklis G, Nikiteas N. Polymorphisms of caspase 8 and caspase 9 gene and colorectal cancer susceptibility and prognosis. Int $\mathrm{J}$ Colorectal Dis. 2011; 26:1113-1118.

45. Umar M, Upadhyay R, Kumar S, Ghoshal UC, Mittal B. CASP8 $-6526 \mathrm{~N}$ del and CASP8 IVS12-19G $>$ A gene polymorphisms and susceptibility/prognosis of ESCC: a case control study in northern Indian population. J Surg Oncol. 2011; 103:716-723.

46. Xiao MS, Zhang DF, Zeng Y, Cheng YF, Yao YG. Polymorphisms in the promoter region of the CASP8 gene are not associated with non-Hodgkin's lymphoma in Chinese patients. Ann Hematol. 2011; 90:1137-1144.

47. George GP, Mandal RK, Kesarwani P, Sankhwar SN, Mandhani A, Mittal RD. Polymorphisms and haplotypes in caspases 8 and 9 genes and risk for prostate cancer: a casecontrol study in cohort of North India. Urol Oncol. 2012; 30:781-789.

48. Hashemi M, Eskandari-Nasab E, Fazaeli A, Rezaei H, Mashhadi MA, Arbabi F, Taheri M. Bi-directional PCR allele-specific amplification (bi-PASA) for detection of caspase-8 $-6526 \mathrm{~N}$ ins/del promoter polymorphism (rs3834129) in breast cancer. Gene. 2012; 505:176-179.

49. Tong N, Zhang L, Sheng X, Wang M, Zhang Z, Fang Y, Xue Y, Li J, Zhang Z. Functional polymorphisms in FAS, FASL and CASP8 genes and risk of childhood acute lymphoblastic leukemia: a case-control study. Leuk Lymphoma. 2012; 53:1360-1366.

50. Wang Q, Yuan Q, Yin C, Zhu J, Qin C, Zhang W. Association of caspases 8-652 $6 \mathrm{~N}$ ins/del polymorphism and risk of renal cell carcmoma in Chinese Han population. Chin J Exp Surg. 2012; 29:2409-2412.

51. Wang YX, Zhao L, Wang XY, Liu CM, Yu SG. Role of Caspase 8, Caspase 9 and Bcl-2 polymorphisms in papillary thyroid carcinoma risk in Han Chinese population. Med Oncol. 2012; 29:2445-2451.

52. de Martino M, Haitel A, Schatzl G, Klingler HC, Klatte T. The CASP8 $-6526 \mathrm{~N}$ insertion/deletion promoter polymorphism is associated with renal cell carcinoma risk and metastasis. J Urol. 2013; 190:717-722. 
53. Wu Z, Li Y, Li S, Zhu L, Li G, Yu Z, Zhao X, Ge J, Cui B, Dong X, Tian S, Hu F, Zhao Y. Association between main Caspase gene polymorphisms and the susceptibility and prognosis of colorectal cancer. Med Oncol. 2013; 30:565.

54. Xiao MS, Chang L, Li WL, Du YS, Pan Y, Zhang DF, Wen Y, Luo J, Li XY, Yao YG. Genetic polymorphisms of the CASP8 gene promoter may not be associated with colorectal cancer in Han Chinese from southwest China. PLoS One. 2013; 8:e67577.

55. Pardini B, Verderio P, Pizzamiglio S, Nici C, Maiorana MV, Naccarati A, Vodickova L, Vymetalkova V, Veneroni S, Daidone MG, Ravagnani F, Bianchi T, Bujanda L, et al. Association between CASP8 $-6526 \mathrm{~N}$ del polymorphism (rs3834129) and colorectal cancer risk: results from a multicentric study. PLoS One. 2014; 9:e85538.

56. Carvalho DC, Wanderley AV, Amador MA, Fernandes MR, Cavalcante GC, Pantoja KB, Mello FA, de Assumpcao PP, Khayat AS, Ribeiro-Dos-Santos A, Santos S, Dos Santos NP. Amerindian genetic ancestry and INDEL polymorphisms associated with susceptibility of childhood B-cell leukemia in an admixed population from the Brazilian Amazon. Leuk Res. 2015; pii: S0145-2126(15)30361-1.

57. Tang YI, Liu Y, Zhao W, Yu T, Yu H. Caspase- 8 polymorphisms and risk of oral squamous cell carcinoma. Exp Ther Med. 2015; 10:2267-2276.

58. de Visser KE, Eichten A, Coussens LM. Paradoxical roles of the immune system during cancer development. Nat Rev Cancer. 2006; 6:24-37.

59. Ho PK, Hawkins CJ. Mammalian initiator apoptotic caspases. FEBS J. 2005; 272:5436-5453.

60. Vogelstein B, Papadopoulos N, Velculescu VE, Zhou S, Diaz LA Jr, Kinzler KW. Cancer genome landscapes. Science. 2013; 339:1546-1558.

61. Tan H, Bao J, Zhou X. Genome-wide mutational spectra analysis reveals significant cancer-specific heterogeneity. Sci Rep. 2015; 5:12566.

62. He J, Liao XY, Zhu JH, Xue WQ, Shen GP, Huang SY, Chen W, Jia WH. Association of MTHFR C677T and A1298C polymorphisms with non-Hodgkin lymphoma susceptibility: evidence from a meta-analysis. Sci Rep. 2014; 4:6159.

63. Xu C, Zhu J, Fu W, Liang Z, Song S, Zhao Y, Lyu L, Zhang A, He J, Duan P. MDM4 rs4245739 A > C polymorphism correlates with reduced overall cancer risk in a metaanalysis of 69477 subjects. Oncotarget. 2016; 7:7171871726. doi: 10.18632/oncotarget.12326.

64. Fu W, Zhuo ZJ, Chen YC, Zhu J, Zhao Z, Jia W, Hu JH, Fu K, Zhu SB, He J, Liu GC. NFKB1 -94insertion/deletion ATTG polymorphism and cancer risk: evidence from 50 case-control studies. Oncotarget. 2017; 8:9806-9822. doi: 10.18632/oncotarget.14190.

65. Xue WQ, He YQ, Zhu JH, Ma JQ, He J, Jia WH. Association of BRCA2 N372H polymorphism with cancer susceptibility: a comprehensive review and meta-analysis. Sci Rep. 2014; 4:6791.

66. He J, Shi TY, Zhu ML, Wang MY, Li QX, Wei QY. Associations of Lys939Gln and Ala499Val polymorphisms of the XPC gene with cancer susceptibility: a meta-analysis. Int J Cancer. 2013; 133:1765-1775.

67. He J, Xi B, Ruiter R, Shi TY, Zhu ML, Wang MY, Li QX, Zhou XY, Qiu LX, Wei QY. Association of LEP G2548A and LEPR Q223R polymorphisms with cancer susceptibility: evidence from a meta-analysis. PLoS One. 2013; 8:e75135.

68. Zhu J, Hua RX, Jiang J, Zhao LQ, Sun X, Luan J, Lang Y, Sun Y, Shang K, Peng S, Ma J. Association studies of ERCC1 polymorphisms with lung cancer susceptibility: a systematic review and meta-analysis. PLoS One. 2014; 9:e97616.

69. He J, Wang F, Zhu JH, Chen W, Cui Z, Jia WH. No association between MTR rs1805087 A $>$ G polymorphism and non-Hodgkin lymphoma susceptibility: evidence from 11486 subjects. Leuk Lymphoma. 2015; 56:763-767.

70. Niu Y, Li F, Tang B, Shi Y, Yu P. Association of hOGG1 Ser326Cys polymorphism with gastric cancer risk: a metaanalysis. Mol Biol Rep. 2012; 39:6563-6568. 